\title{
On the Resolution of Chaos in Population Models
}

\author{
JOHN VANDERMEER \\ Division of Biological Sciences, University of Michigan, Ann Arbor, Michigan 48109 \\ Received February 25, 1980
}

\section{INTRODUCTION}

Some simple equations representing the dynamics of a single population over time are known to be capable of generating extremely complicated behavior. This complicated behavior has been termed chaotic ( $\mathrm{Li}$ and Yorke, 1975; May, 1974, 1975; May and Oster, 1976; Guckenheimer et al., 1976). The practical consequence of chaotic behavior is a certain inability to describe particular population trajectories with any degree of accuracy. The purpose of this communication is to demonstrate that for certain models, when properly viewed, extremely chaotic populations can behave in very regular and predictable ways, similar to that noted by May (1975). Furthermore, it appears that, in a sense, the more chaotic a population, the more predictable its behavior, at least for a certain class of models.

\section{Regular Patterns in Chaotic Behavior}

In all that follows we shall be working with the equation

$$
X(t+1)=F(X(t))
$$

where $X(t)$ is population density at time $t$, and we suppose that $F$ has only one local maximum. If we symbolize $F^{n}$ as the $n$th composition of $F$ (i.e., $F^{n}(X)=F\left(F^{n-1}(X)\right)$, we can define several important regions of $X$ space. Let $k_{1}$ be a value of $X(t)$ for which $F$ is the maximum. We now define $k_{2}=$ $F^{-2}\left(k_{1}\right)$. Let $k_{3}$ be the value of $X(t)$ for which $X(t)=F(X(t))$ (i.e., the point at which the function crosses the $45^{\circ}$ line). Define $k_{4}=F^{-1}\left(k_{3}\right)$. Finally, let $k_{5}$ be the value of $X$ that generates $k_{2}$, i.e., $k_{2}=F\left(k_{5}\right)$. Thus we have the following sets: $\quad \alpha=\left\{X \mid 0 \leqslant X<k_{2}\right\}, \quad \beta=\left\{X \mid k \leqslant X<k_{4}\right\}, \quad \gamma_{1}=$ $\left\{X \mid k_{4} \leqslant X<k_{1}\right\}, \quad \gamma_{2}=\left\{X \mid k_{1} \leqslant X<k_{3}\right\}, \quad \gamma_{3}=\left\{X \mid k_{3} \leqslant X<k_{5}\right\}$, and $\Delta=$ $\left\{X \mid X>k_{5}\right\}$. An example of an $F$ along with the boundaries of the various sets is shown in Fig. 1a, and a diagram of the necessary dynamics of that 


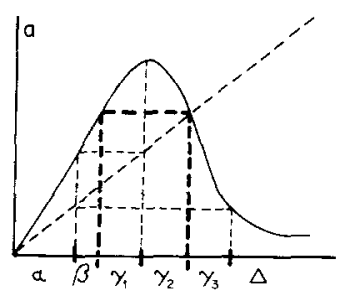

b

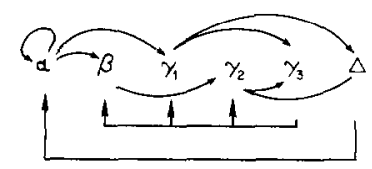

FIG. 1. (a) An example of a function for Eq. (1) in which the various sets refered to in the text have been illustrated. (b) The necessary dynamical behavior of the function from (a).

function is presented in Fig. Ib. From Fig. $1 \mathrm{~b}$ we can construct a transition matrix (Guckenheimer et al., 1976) as

\begin{tabular}{l|cccccc}
\multicolumn{1}{c}{} & \multicolumn{1}{c}{$\alpha$} & $\beta$ & $\gamma_{1}$ & $\gamma_{2}$ & $\gamma_{3}$ & $\Delta$ \\
\cline { 2 - 7 }$\alpha$ & $P_{11}$ & $P_{12}$ & $P_{13}$ & 0 & 0 & 0 \\
$\beta$ & 0 & 0 & 0 & 1 & 0 & 0 \\
$\gamma_{1}$ & 0 & 0 & 0 & 0 & $P_{35}$ & $P_{36}$ \\
$\gamma_{2}$ & 0 & 0 & 0 & 0 & $P_{45}$ & $P_{46}$ \\
$\gamma_{3}$ & 0 & $P_{52}$ & $P_{53}$ & $P_{54}$ & 0 & 0 \\
$\Delta$ & 1 & 0 & 0 & 0 & 0 & 0
\end{tabular}

The conditions of this matrix dictate what sorts of qualitative bchavior the model will exhibit. Specifically, if $P_{36}=P_{46}=0$, chaotic behavior is not possible (this fact follows elementarily from the $\mathrm{Li}-$ Yorke theorem). But the most important thing to note about the above matrix is that the only region that can be mapped onto itself is the set $\alpha$. If $P_{11}$ is very large, the population could stay in this region for a relatively long period of time before going on to $\beta$ or $\gamma_{1}$. For all other regions in $X(t)$, exit is mandatory after a single time period.

Relatively speaking, the set of points $\alpha$ represents population densities that can be called rare. At the other extreme, points in $\Delta$ represent population densities that can be called abundant. It is evident from the above matrix that any population in $\Delta$ at time $t$ must return to $\alpha$ at time $t+1$, and furthermore, no population can ever enter $\Delta$ unless it is a chaotic population. 
Finally we note that access to $\alpha$ is limited to those populations that are already in $\alpha$, or are in $\Delta$. Thus, once a non-chaotic population leaves $\alpha$, it never returns. Non-chaotic populations will never be rare (in the sense that rare is defined by the set $\alpha$ ).

The above qualitative observations lead to an obvious consequence and an interesting speculation. The obvious consequence is that in a chaotic population every population flush must be followed by a period of rarity. The interesting speculation is that the population flushes and the periods of rarity may be somehow related in a regular way. If such a speculation is true, it will point to a certain regularity in chaotic populations.

We examine this speculation first by assuming specific forms for $F$ in the two regions, $\alpha$ and $\Delta$. Assume that for all $X \in \alpha, X(t+1)=a X(t)$ (i.e., at low population densities the population grows exponentially), and for all $X \in \alpha, X(t+1)=e^{-b X(t)}$ (i.e., at very high densities the population overexploits its resources at an exponentially decreasing rate). Beginning with some value of $X(t),\left(X_{0}\right)$, we can compute how many time periods it will take for the population to reach the upper boundary of $\alpha, k_{2}$. That value is given as

$$
n=\left(\ln k_{2}-\ln X_{0}\right) / \ln a
$$

The value that would have generated $X_{0}$ from the set $\Delta$ is given as

$$
X_{0}=e^{-b X_{\mathrm{F}}}
$$

where $X_{\mathrm{F}}$ symbolizes the size of the population at its flush. The above two equations combine to give

$$
n=\ln \left(k_{2}-a\right)+\left(b / \ln \text { a) } X_{\mathrm{F}} .\right.
$$

Thus we see that the length of time the population will be rare, following a population flush is expected to be linearly related to the size of the population flush, under the specific hypotheses of exponential increase in $\alpha$ and exponential decay in $\Delta$.

More generally, we apply simple linear approximations in both $\alpha$ and $\Delta$ sets. Thus

$$
n=C_{1}-\frac{\ln \left(C_{2}-C_{3} X_{\mathrm{F}}\right)}{\ln a}
$$

and

$$
\frac{\partial n}{\partial x}=\frac{C_{3}}{\left(C_{2}-C_{3} X_{F}\right) \ln a}
$$

Thus we see that over the range of interest $\left(C_{2}-C_{3} X>0\right)$, the time spent being rare $(n)$ is positively related to the size of the population flush. 
This result suggests a sort of qualitative conservation law. The mean population density will be constrained within certain narrow limits since a large population flush will be followed by a relatively lengthy period of rarity and a smaller population flush will be followed by a relatively short period of rarity.

\section{A Biological Example}

In an earlier paper (Vandermeer, 1975) I outlined a descrite equation approach to the description of seed predation by insects, in which a recruitment function $f(x)$ stipulated next season's number of individual insects attacking seeds as a function of $x$, the number attacking the seeds this year. This recruitment function was linked to an attack function $g(x)$ and the boundary conditions $\partial f / \partial x>0$ for $x$ small, $\partial f / \partial x \gtreqless x$ for $x$ large, and $\partial q / \partial x>0$, and $\partial^{2} g / \partial x^{2}>0$. Subsequent work on chaos has made it obvious that according to my model, the insect population will be chaotic if $\partial f / \partial x$ is small enough for large $x$ (i.e., large absolute value with negative sign) or $\partial g / \partial x$ is large enough for large $x$-specifically for the non-zero value of $x$ at which $f$ and $g$ are equal.

The attack rate function can easily be incorporated into the recruitment function so as to express the model in the more usual form of Eq. (1).

There are particular biological features of insect seed predation that make it possible to stipulate $F$ directly in terms of certain probabilities. Let $b$ symbolize the average number of eggs laid by an individual insect. Supposing that the eggs are laid at random, the fraction of seeds that are attacked by a single larvae is given by the second term of a Poisson series, or

$$
P(1)=(b x / m) e^{-b x / m}
$$

where $m$ is the total number of seeds (thus $b x / m$ is the mean number of eggs per seed). We now presume that any seed attacked by more than one larvae will not offer enough resource to provide sustenance for both larvae and thus neither will reach the pupation stage. Obviously this assumption could easily be relaxed by taking more terms in the Poisson series. I here utilize only the single term for ease of exposition.

Thus, the number of individual insects expected in the next generation can be written as

$$
X(t+1)=F(X(t))=a b X(t) e^{-b X(t) / m}
$$

where $a$ is the survivorship rate. This form corresponds to May's form $B$ (May, 1976) where the carrying capacity $K$ is $m(\ln a b) / b$ and growth rate $r$ 


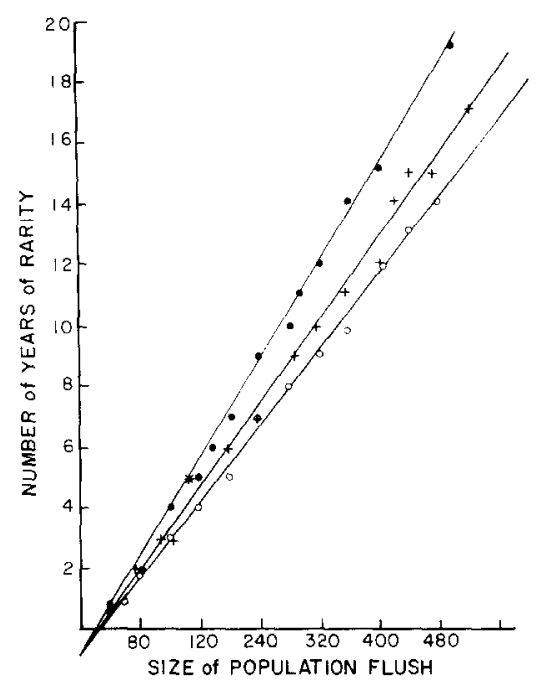

FIG. 2. The relationship between peak population density and subsequent years of rarity for various parameter values of Eq. (2) $(\bullet, a b=125 ; \times, a b=250 ; O, a b=500)$.

is $\ln a b$. Thus we can catalogue the population behavior as asymptotic equilibrium if $a b<7.39$, as oscillatory (with various periods) if $7.39<$ $a b<14.73$, and as chaotic if $a b>14.73$ (May, 1976; May and Oster, 1976).

In Fig. 2 is plotted the relationship of $n$ versus peak population density for increasing values of $r$ for Eq. (2). Note that the slope decreases as $r$ is increased; that is, as the population becomes more and more chaotic, less time is spent in rarity as a function of particular population flushes. Furthermore, as the population becomes excessively chaotic ( $r$ becomes very large), the change in the slope of the relationship between $n$ and $X_{F}$ decreases. At very large parameter values of $r$ ("super" chaos), the relationship between $n$ and $X_{\mathrm{F}}$ changes only slightly with a change in $r$. This is an interesting result because along with the regular behavior of $n$ versus $X_{\mathrm{F}}$ it implies a certain resolution of chaotic behavior. To recapitulate we first noted that the number of years of rarity is tightly correlated with the size of the preceeding population flush. We now see that under conditions of extreme chaos the correlation itself becomes almost invariable. Thus, from a practical point of view the number of years of rarity of a population can be generally predicted from a knowledge of the size of a population flush. If the population is only "mildly" chaotic (e.g., the functions toward the left of Fig. 2), slight changes in $r$, as might happen as a result of typical stochastic forces, will cause a dramatic drop in the accuracy of such predictions. But in a superchaotic population (e.g., the functions toward the right of Fig. 2) small changes in $r$, for whatever reason, will have little effect on the ability 
to predict $n$ from $X_{\mathrm{F}}$. The chaos has been, in a sense, resolved. This resolved behavior is reminiscent of patterns obtained by May in his study of differential-delay equations (May, personal communication), where regular oscillations are replaced by seemingly chaotic behavior which is subsequently replaced by regular oscillations again, in response to a single varying parameter.

\section{Resolved Chaos}

We now consider a restricted class of functions for $F$; namely, we are concerned with that class of which $\partial F / \partial X(t)<0$ for large values of $X(t)$. Biologically, the concern is with those situations in which overexploitation is a possibility.

Given such functions, the population can be pulsed artificially, whether or not the parameters dictate chaotic behavior. That is, for a non-chaotic population it is impossible for the population ever to reach the region $\Delta$. But it is possible to begin a trajectory in $\Delta$ simply by starting the population there. If we assume, as before, that $F$ is approximately linear in both $\alpha$ and $\Delta$, with positive slope in $\alpha$ and negative slope in $\Delta$, the analysis as presented earlier applies directly. There will be a regular and positive relationship between the size of the artificially induced flush and the number of the time units of rarity to follow. This means that a heretofore unrecognized regularity exists for non-chaotic and chaotic populations alike; that is, the size of a population flush (naturally occurring in a chaotic population, artificially induced in a non-chaotic population) predicts the length of time the population will subsequently remain in a condition of rarity. Furthermore, as will be suggested in the following example, that regularity itself shows predictable behavior, sometimes transforming the typically unpredictable behavior of a chaotic population into a highly predictable form but retaining its underlying chaotic form. It is this region of high predictability that I call resolved chaos.

Recall the seed predator example given earlier. Pulsing the population artificially we obtain the data plotted in Fig. 3. It is obvious that the same positive relationship exists between peak population density and subsequent time being rare, as existed in chaotic populations. The difference, of course, is that the relationship cannot in principle be observed in nature for nonchaotic populations whereas it can for chaotic ones.

We can summarize the information in Figs 2 and 3 by plotting a graph of the slope of the line (in Figs. 2 and 3) against the parameter of the equation. Such a graph is presented in Fig. 4. It is interesting that the various forms of population behavior from asymptotic stability, to damped oscillations, to permanent oscillations to chaos, can be visualized on this single graph. It 


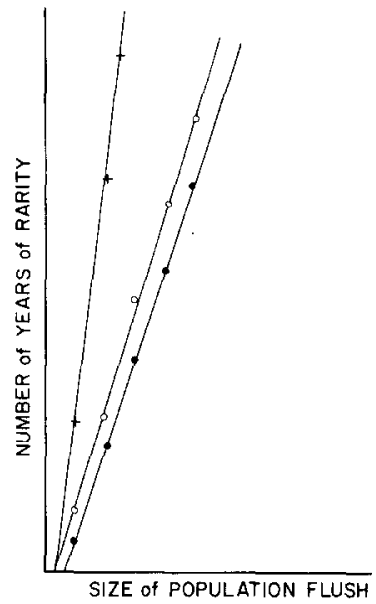

FIG. 3. The relationship between peak population density and subsequence years of rarity for various parameter values of Eq. (2). All examples are artificially pulsed non-chaotic or midly chaotic populations $(\bullet, a b=14.73 ; 0, a b=10 ; \times, a b=2.5)$.

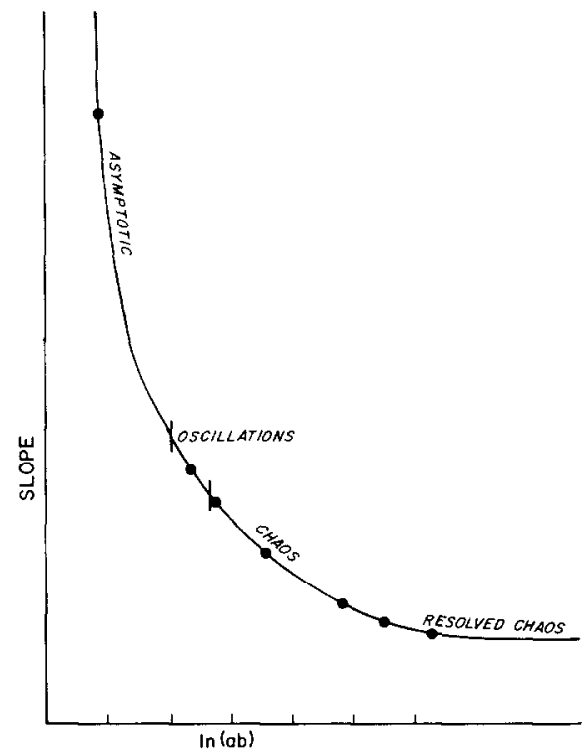

FIG. 4. The relationship between the slope of the "population flush versus time of rarity" function and the parameter of Eq. (2). 
should be recalled, however, that the only potentially observable points are those in the chaotic regime.

Figure 4 represents a compact summary of possible qualitative population behaviors. But the relation in Fig. 4 is derived from a particular equation. Whether or not the same sort of relationship will exists for other equations is not known. It is clear that the descending limb of $F$ must at least have a positive second derivative $\left(\partial^{2} F / \partial X^{2}>0\right)$ for the relationship pictured in Fig. 4 even to hold approximately.

As a counterexample consider the triangular function (as in Guckenheimer et al., 1976)

$$
\begin{array}{ll}
X(t+1)=a X(t) & \text { for } \quad X \in \alpha \\
X(t+1)=b-c X(t) & \text { for } \quad X \in \Delta
\end{array}
$$

As before, we have

$$
n-\left(\ln k_{2}-\ln X_{0}\right) / \ln a
$$

and $X_{0}$ is given as

$$
X_{0}=b-c X_{\mathrm{F}}
$$

So

$$
n=\frac{\ln k_{2}}{\ln a}-\frac{\ln \left(b-c X_{\mathrm{F}}\right)}{\ln a}
$$

and, as before,

$$
\frac{\partial n}{\partial x}=\frac{c}{\left(b-c X_{\mathrm{F}}\right) \ln a}
$$

In this particular case, $c$ is the parameter that determines chaos-as $c$ gets larger the population is pushed further and further into the chaotic regime. We are interested in the rate of change of this slope as a function of the parameter $c$, namely,

$$
\frac{\partial^{2} n}{\partial x \partial c}=\frac{b}{\left(b-c X_{\mathrm{F}}\right)^{2} \ln a}
$$

which will always be positive. Such a result is in direct contradiction to the general pattern described earlier for the specific equation (2).

But it is also obvious from Eq. (3) that $\partial n / \partial x$ will be negatively related to chaos. In Fig. 5 I have illustrated the general form of this relationship with piecewise linear forms of $f$, for $\partial n / \partial x$ positive and negative (we note in 

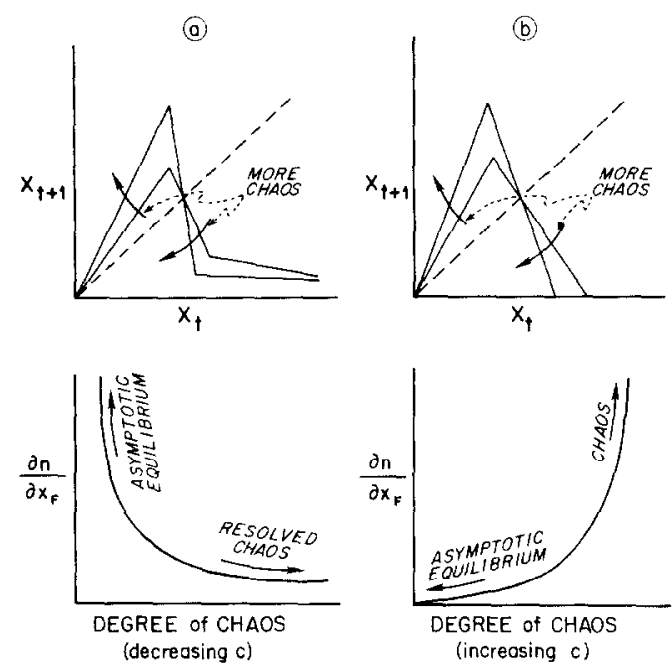

FIG. 5. Illustration of the general form of $F$ and the possibility of resolving chaotic patterns. Examples where chaos (a) will be resolved and (b) will not be resolved.

passing that $\partial^{3} n / \partial x \partial c^{2}=b X_{\mathrm{F}}\left(b-c X_{\mathrm{F}}\right)^{-3}(\ln a)^{-1}$ which is negative only when $c X>b$, a situation out of the realm of interest for this paper). In Fig. 5 we see an interesting limitation on the resolution of chaos. Apparently some functions (e.g., Fig. 5b) will not generate resolved chaos, while others (e.g., Fig. 5a) will generate resolved chaos. Furthermore, it appears that if the descending limb of $F$ has an inflection point (i.e., $\partial^{2} F / \partial X^{2}=0$ for some $X$ on the descending limb), resolution of chaos is possible, whereas if $F$ has no inflection point in its descending limb, resolution of chaos is impossible.

\section{Discussion}

Three mathematical considerations have been presented: (1) the number of time units a population will remain rare is a monotonically increasing function of the size of the previous population flush, (2) the slope of that function follows a regular pattern as propensity for chaotic behavior is increased, sometimes leading to a region in which further changes in propensity for chaos result in very small changes in the slope-this region is called the region of resolved chaos, and (3) the resolution of chaos is possible only if $\partial^{2} F / \partial X^{2}=0$ for some $X \in\left(\gamma_{2} \cup \gamma_{3} \cup \Delta\right)$. Each of these three considerations suggests biological consequences that need to be discussed.

The relationship between time of rarity and size of previous population flush is an important consequence of chaos. It is especially interesting in 
light of the fact that only in the chaotic regime does the relationship hold. We might then suggest that observing such a relationship under natural conditions would be evidence that the population of concern was in fact exhibiting chaotic behavior.

The general pattern further suggests that chaotic populations are likely to be usually rare. While population flushes occur occasionally, the time between flushes is time in which the population is rare. Since rare populations are not popular subjects for biological study, it is perhaps not surprising that the detection of chaos in nature. has been thus far rather elusive (Hassel et al. (1976).

The second consideration relates to the changes to be expected in the pattern described above, as a population becomes more and more chaotic. For chaotic populations the number of time units of rarity is closely related to the size of the previous population flush. But the nature of that relationship changes as the population becomes more and more chaotic. And, for a broad class of models, when the population approaches extreme chaos, the degree to which that relationship changes becomes very small. Thus, for example, for a "mildly" chaotic population one could theoretically predict how many years the population will remain rare after a population flush of a particular size. But if there is any sort of variation in the environment (e.g., stochastic variation), the accuracy of the prediction will be dramatically affected. Yet, if a similar population were "very" chaotic, similar variation would not affect the accuracy of the prediction. In a sense the chaos has been resolved. The population, properly viewed, is highly predictable and that predictability is relatively resistant to outside perturbation.

Not all models which can exhibit chaos can also exhibit resolved chaos. Apparently it is the details of the region of overexploitation that determines whether or not chaos will be resolved as chaotic behavior is increased. Unfortunately those details defy a nice intuitive summary. If the descending limb of $F$ has an inflection point, chaos is resolvable. If the descending limb of $F$ does not have an inflection point, chaos is not resolvable. But exactly what does that mean in biological terms? While a clearcut intuitive description does not seem possible, a sort of law of diminishing returns seems appropriate. If the addition of more and more individuals into an already overcrowded population reaches a limiting value in terms of the negative-density-dependent effects it induces, that is, if the addition of one more individual to an extremely overcrowded situation would add less negative feedback than if that same individual had been added to a mildly overcrowded situation, the chaos of the model can be resolved.

While not diminishing the potential importance of chaotic behavior in nature, the notions of regular patterns and resolved chaos may provide a new technique for dealing with empirical data. For those populations whose 
behavior over time defies regular description, looking at the relationship between peak population density and period of rarity may provide insight as to the underlying dynamics of the population, particularly with regard to the refinements of chaos theory presented herein.

\section{REFERENCES}

Guckenheimer, J., Oster, G. F., AND Ipaktchi, A. 1976. The dynamics of density dependent models, Math. Biol.

Hassell, M. P., Lawton, J. H., and May, R. M. 1976, Patterns of dynamical behaviour in single-species populations, J. Anim. Ecol. 45,

LI, T. Y., And Yorke, J. A. 1975. Period three implies chaos. Amer. Math. Monthly 82. 985-992.

MAY, R. M. 1974. Biological populations with nonoverlapping generations: Stable points, stable cycles, and chaos, Science 186, 645-647.

MAY, R. M. 1975. Biological populations obeying difference equations: Stable points, stable cyles, and chaos. J. Theor. Biol. 49, 511-524.

MAY, R. M. 1976. Models for single populations, in "Theoretical Ecology Principles and Applications" (May R. M., ed.), pp. 4-25, Saunders, Philadelphia, Pa.

MAY, R. M., AND OSTER, G. F. 1976. Bifurcations and dynamic complexity in simple ecological models, Amer. Natur. 110 ,

VANDERmeer, J. H. 1975. A graphical model of insect seed predation, Amer. Natur. 109. $147-160$. 